\title{
Swelling of Thin Poly(ethylene glycol)-Containing Hydrogel Films it Water Vapor-A Neutron Reflectivity Study
}

Thomas Ederth and Tobias Ekblad

The self-archived postprint version of this journal article is available at Linköping University Institutional Repository (DiVA):

http:// urn.kb.se/ resolve?urn=urn:nbn:se:liu:diva- 148654

N.B.: When citing this work, cite the original publication.

Ederth, T., Ekblad, T., (2018), Swelling of Thin Poly(ethylene glycol)-Containing Hydrogel Films it Water Vapor-A Neutron Reflectivity Study, Langmuir, 34(19), 5517-5526.

https:// doi.org/ 10.1021/ acslangmuir.8b0017

Original publication available at:

https:/ / doi.org/ 10.1021/ acs.langmuir.8b00177

Copyright: American Chemical Society

http:// pubs.acs.org/ 


\title{
Swelling of thin poly(ethylene
}

\section{glycol)-containing hydrogel films in water vapour - A neutron reflectivity study}

\author{
Thomas Ederth ${ }^{* \dagger \dagger}$ and Tobias Ekblad ${ }^{\dagger, \dagger}$ \\ $\dagger$ Division of Molecular Physics, Department of Physics, Chemistry and Biology, Linköping \\ University, SE-581 83 Linköping, Sweden \\ $\ddagger$ Present address: MariboHilleshög Research AB, Landskrona, Sweden \\ E-mail: ted@ifm.liu.se
}

\begin{abstract}
Hydrogels are widely used in biomedicine and for bioanalytical purposes, normally under wet conditions. For certain applications, processing steps or process monitoring, hydrogel films are used or treated under ambient conditions, and since they are hygroscopic, it is of interest to investigate how they respond to changes in atmospheric humidity. We have used neutron reflectometry to follow the swelling of thin UV-polymerized hydrogel films in air under different relative humidities. These polymers were prepared to similar thicknesses on silica and gold substrates, and the chemical similarity between them was verified by infrared spectroscopy. The swelling in response to variations in relative humidity was different for the layers on the two substrate types, reflecting structural changes induced by differences in the UV exposure required to achieve a given polymer thickness, as demonstrated also by differences in the Flory-Huggins interaction parameter, obtained by fitting a Flory-Huggins-type sorption model to the swelling
\end{abstract}


data. Wetting studies show small changes in contact angles with surrounding humidity variations, indicating that structural reorganization at the interface in response to humidity changes are limited.

\section{Introduction}

Hydrogels are highly water-soluble polymer networks, which may be made from a variety of natural (e.g. saccharide, protein) or synthetic (e.g. ethylene oxide, acrylamide) polymers. ${ }^{1}$ The high water content gives hydrogels properties which are in some respects similar to biological tissue, and as a result of considerable flexibility in chemical ${ }^{2,3}$ and structural ${ }^{4}$ properties, and the use of responsive materials, ${ }^{5}$ they have found widespread use in biomaterials science and medicine, in applications such as contact lenses, implants, wound dressings, controlled drug release or tissue engineering. ${ }^{6,7}$ Many hydrogels show very low non-specific adsorption of proteins, and are used as surface coatings to promote biocompatibility. ${ }^{8}$ Requirements on biodegradeability, the use of biomolecular self-assembly, and the incorporation of drugs have stimulated research in hydrogels based on biological materials, such as silk proteins, ${ }^{9}$ plasmid DNA ${ }^{10}$ or peptides. ${ }^{11}$

Hydrogels are also used for a variety of sensing applications. ${ }^{12}$ Beside biosensors, ${ }^{13}$ we find, for example, ion, ${ }^{14} \mathrm{pH},{ }^{15}$ oxygen,${ }^{16}$ humidity ${ }^{17}$ and ethanol vapour ${ }^{18}$ sensors. In biosensor applications, where surface-sensitive detection methods such as ellipsometry or surface plasmon resonance (SPR) are used, hydrogels act as surface-enlarging matrices into which ligands, ranging from small molecules to proteins and ultimately to cells, are immobilized, ${ }^{19}$ allowing the number of adsorbed ligands per surface area to be increased far beyond the equivalent of a monolayer. Carboxymethylated dextran matrices have been extensively used for this purpose ${ }^{20}$ but suffer from relatively high nonspecific binding in complex biological fluids such as blood serum, plasma, or cell lysates, and materials based on poly(ethylene glycol) (PEG) (or oligo(ethylene glycol), OEG) have long been considered promising alternatives. The use of PEG-containing coatings to reduce protein adsorption ${ }^{21}$ and to enhance 
biocompatibility ${ }^{22}$ are well established, and other uses, e.g. to reduce platelet adhesion ${ }^{23}$ or bacterial infections ${ }^{24}$ have been added more recently. The limited chemical stability of endgrafted PEG brushes makes these unsuitable for long-term applications, and PEG-containing hydrogels with more stable backbones are considered instead. ${ }^{25,26}$ As a model for these, we use PEG-based hydrogel matrices graft co-polymerized from substrates from a mixture of 2hydroxyethyl methacrylate (HEMA) and hydroxyl-terminated PEG methacrylate monomers with on average 10 ethylene glycol units in the chain $\left(\mathrm{PEG}_{10} \mathrm{MA}\right)$, using a UV-initiated free radical reaction, self-initiated photografting and photopolymerization (SIPGP). The resulting thin hydrogel films show excellent resistance to non-specific adsorption from fibrinogen solutions, as well as from blood plasma and serum. ${ }^{25}$ The possibility to further modify these to contain carboxylic groups, allowing ligand immobilization in a controlled and functional manner, indicates that this PEG-containing matrix is suitable for biochip and biosensor applications in demanding biofluids. ${ }^{25}$ Synthetic routes to facilitate growth of these hydrogels onto polymers, glass/silica and other oxides (via organosilanes) as well as gold and other metals (via thiol chemistry), permits considerable flexibility in the choice of substrate. ${ }^{27}$ Processing methods to prepare hydrogel gradients ${ }^{28}$ and patterns, ${ }^{27}$ further show the potential of these materials both for practical biochip applications, ${ }^{29}$ and as versatile tools for exploring fundamental aspects of fouling resistance or interactions in polymer systems. ${ }^{30}$ SIPGP-prepared hydrogels also reduce cell adhesion, ${ }^{31}$ and we have demonstrated that these hydrogels show excellent stability and antifouling properties in marine biofouling tests, using common fouling organisms in laboratory assays. ${ }^{32}$

In most practical applications, hydrogels are used in aqueous environments, where properties such as the swollen thickness, the polymer chain segment density distribution, or the penetration depth of immobilized ligands or analytes, are of importance. However, much of the process monitoring, modification and characterization during preparation of these matrices is carried out in ambient atmosphere. Since these are hygroscopic materials, it is of considerable interest to study the swelling of the hydrogel under varying atmospheric 
humidities, to establish the effects of humidity on the characterization data. This refers to parameters such as the layer thickness (or the polymer mass per surface area), lateral homogeneity and wettability, and the dependence of these on process parameters. Further, PEG and PEG-containing layers have been used in a variety of humidity sensors, based on dimensional, ${ }^{33}$ electrical, ${ }^{34}$ mass $^{35}$ or refractive index ${ }^{36}$ changes upon swelling, and there is an interest in the ability to tune the polymer properties to optimize sensing performance to different applications. Hence, control of the water uptake characteristics of PEG-based polymers supports the informed and systematic development of such sensors. The wetting of water-soluble polymers by aqueous droplets depend on the ambient humidity, ${ }^{37}$ and while this is a topic of great fundamental interest, a both relevant and important application is the spreading of microdroplets onto hydrogels during the printing of biomolecules in biochip production, ${ }^{38}$ which is also an application area where PEG hydrogels are utilized.

The choice of substrate for the hydrogel is not without significance; we have observed that the UV-initiated growth of hydrogels on silica (via a silane linker layer) and gold (via a thiol layer) proceeds at very different rates; the reason for this is not clear, but it appears that these different chemistries result in different graft densities, ${ }^{39}$ and a difference in structure may be of relevance to sensing applications. Although silica interfaces occur in numerous bioanalytical and microelectromechanical devices and applications, metal substrates are required for SPR detection, where gold is usually the preferred metal, and also the substrate for the hydrogel-containing sensing layer. Gold electrodes are also dominating in Quartz Crystal Microbalance (QCM) sensors. Thus, both gold and silicon substrates are widely used for bioanalytical purposes, and it is of interest to investigate differences in hydrogels grafted onto silica and gold substrates under identical conditions.

Structural characterization of a swollen (wet) hydrogel is difficult due to the dilute character of the polymer and the low optical and X-ray contrasts between water and the often hydrogen- and oxygen-rich hydrogel, requiring extensive spectroscopic ellipsometric (SE) studies, or the contrast provided by deuterium labelling in neutron reflectometry (NR) exper- 
iments. The much enhanced contrast obtained by $\mathrm{H} \rightarrow \mathrm{D}$-substitution in neutron scattering is an important tool for polymer science. Neutron reflectometry in particular, besides being of general use for studying soft matter at interfaces ${ }^{40}$ can provide polymer chain segment density profiles of polymers at interfaces. For example, structural studies of OEG methacrylate polymers ${ }^{41}$ and end-grafted PEG layers ${ }^{42}$ have been used to explore details about protein resistance mechanisms, and also the interactions of proteins with brushes. ${ }^{43}$ Among recent uses of NR for structural characterization of polymers, there are also studies carried out under different vapours; Müller-Buschbaum et al. have studied various copolymers of $\operatorname{poly}(N-$ isopropylacrylamide) ${ }^{44-46}$ and poly(methoxydiethylenglycol acrylate) ${ }^{47,48}$ under humid conditions, Galvin and Genzer et al. used both SE and NR for studies of polyelectrolyte brushes in water and alcohol vapours, $;^{49,50}$ other recent work concern the swelling of polystyrene brushes in toluene vapour, ${ }^{51}$ and the effect of water vapour on polyelectrolyte multilayers. ${ }^{52}$

Here we report on the structural characterization of swollen HEMA-co-PEG ${ }_{10}$ MA hydrogels grafted from silica and gold substrates via SIPGP, in humid atmosphere, using neutron reflectometry. Taking advantage of the contrast provided by $\mathrm{H} \rightarrow \mathrm{D}$ isotopic substitution of the water penetrating into the polymer matrix, we have determined the change in water content and polymer layer thickness upon increasing atmospheric humidity. Further, the wettabilities of the polymers have been investigated over a wide range of humidities, and the chemical structure monitored by infrared spectroscopy.

\section{Experimental section}

\section{Materials}

Materials: HEMA and $\mathrm{PEG}_{10} \mathrm{MA}\left(\mathrm{M}_{n} \approx 500\right.$, ca. 10 PEG units $)$ were purchased from Sigma-Aldrich, $\gamma$-methacryloxypropyltrimethoxysilane (MPS, sold under the trade name PlusOne $^{\mathrm{TM}}$ Bind-Silane) was purchased from GE Healthcare Life Sciences, Sweden. Glacial acetic acid was obtained from Merck KGaA, Germany. Titanium was 99.9\% (Balzers), and 
gold 99.99\% (Nordic High Vacuum AB, Sweden). 16-thiohexadecanol ( $\geq 99.5 \%)$ was a gift from Biacore AB (now GE Healthcare). All chemicals were used as received.

\section{Hydrogel preparation}

The hydrogels were prepared on pieces of polished silicon wafers, or on silicon blocks $(50 \times$ $50 \times 10 \mathrm{~mm}^{3}$ ) for neutron reflectometry, either on the native oxide via a silane linker layer, or on thin gold films deposited on the oxide. Before use, the silicon substrates were cleaned in TL1 solution (1:1:5 ratio of $25 \% \mathrm{NH}_{3}, 30 \% \mathrm{H}_{2} \mathrm{O}_{2}$ and MilliQ water for 10 min at $85^{\circ} \mathrm{C}$ ). For silanization, blocks were immersed in a 1:1 mixture of ethanol and MilliQ water containing 0.4\% MPS and $0.05 \%$ glacial acetic acid. After 10 minutes, the blocks were removed from the silane solution and dried under a stream of nitrogen gas, before the silane layer was cured in an oven at $115{ }^{\circ} \mathrm{C}$ for 10 minutes. To remove any silane multilayers, the blocks were ultrasonicated in ethanol for 10 seconds, further rinsed with ethanol and dried. For gold coating, substrates were mounted in an electron-beam UHV evaporation system. Deposition of a 1-nm titanium adhesion layer preceded a 10-nm gold layer. Evaporation rates were set to 0.1 and $0.5 \mathrm{~nm} / \mathrm{s}$ for $\mathrm{Ti}$ and $\mathrm{Au}$, respectively. The base pressure was typically below $5 \times 10^{-9}$ Torr before evaporation started, and the pressure during the gold evaporation step $\leq 5 \times 10^{-8}$ Torr. The coated substrates were then stored in sealed containers until further use. Before hydrogel grafting, they were TL1-cleaned again, and immersed in a $1 \mathrm{mM}$ solution of 16-thiohexadecanol in ethanol overnight, whereafter they were sonicated in ethanol for 2 minutes to remove physisorbed thiols, rinsed with ethanol and dried.

The hydrogel coatings were prepared by polymerization onto the silanized or thiolated substrates, respectively, and the two types of substrates were treated identically from this point. The monomer solution consisted of $120 \mathrm{mM}$ HEMA and $120 \mathrm{mM} \mathrm{PEG}_{10} \mathrm{MA}$ dissolved in MilliQ water. No initiator was added and the monomers were used without purification. The polymerization process and the reactor setup have been described in detail elsewhere, ${ }^{25}$ and is described only briefly in the following. A sandwich of a UV-transparent quartz disc, the 
monomer solution and the substrate was constructed by applying $5 \mu \mathrm{l}$ of monomer solution per $\mathrm{cm}^{2}$ substrate area on the face of the substrate, and gently putting the quartz plate on top. The sandwich was then placed under a UV lamp with the main emission peak at 254 nm (Philips TUV PL-L, 18W). The irradiation time was $10 \mathrm{~min}$ for the hydrogels grown onto silanized silica, and $3 \mathrm{~min}$ for the hydrogels on the gold-coated samples; the growth rate is faster on gold substrates, and these two exposure times typically result in films of similar (dry) ellipsometric thicknesses. The blocks were then removed and ultrasonicated in ethanol and water, thoroughly rinsed with ethanol and dried. Advancing contact angles were measured on the silanized silicon surface and on the finished hydrogels to verify the consistency of the preparation procedure; from past experience the acceptable span is set to 55-64 ${ }^{\circ}$. Control experiments confirm grafting from both the methacrylate-terminated MPS and the OH-functionalized thiol (but not to gold without the alkylthiol layer), and with polymer layer thicknesses on both substrates unchanged after sonication in water, ethanol or dichloromethane.

\section{Contact angle measurements}

Advancing and receding angles of water were measured under controlled humidity conditions with a KSV CAM200 contact angle meter (KSV, Helsinki, Finland). The measurements were carried out in a Ramé-Hart 100-07 Environmental Chamber (Ramé-Hart, NJ, USA) with a syringe mounted above and the needle inserted through a membrane. Humidity was measured with a Honeywell HIH-4000 sensor mounted within $2 \mathrm{~cm}$ from the sessile droplet. Humidity was adjusted by mixing dry nitrogen gas with nitrogen which has been humidified by passing two gas wash bottles filled with water, where the second was heated to approx $50^{\circ} \mathrm{C}$. The exit tube passed a liquid trap to prevent condensation water from entering the measurement chamber. All measurements were conducted from lower to higher humidity. Advancing angles were measured by slowly expanding a sessile droplet via the screw-controlled syringe so that the three-phase line moved over the surface, and taking 
photographs of the droplet during this expansion. Analysis of the thus obtained images yielded two contact angles per image, and each data point represents the average of two angles each from ten images. Receding angles were determined similarly, but while withdrawing liquid from the sessile droplet.

\section{FTIR-ATR}

Hydrogels of the two sample types were examined by FTIR-ATR using a PIKE MIRacle single-reflection ATR accessory, with a diamond-coated ZnSe prism. Data was collected with a Bruker Vertex 70 spectrometer using an MCT detector, at a resolution of $4 \mathrm{~cm}^{-1}$, by adding 3000 scans for each measurement. TL1-cleaned silicon or gold-coated silicon substrates without hydrogels were used as references to obtain background spectra. Spectra were baseline-corrected using a 5-point concave rubberband method.

\section{Neutron reflectometry}

The neutron reflectivity experiments were performed on the fixed-wavelength $(\lambda=4.41 \AA)$ reflectometer ADAM at the Institute Laue-Langevin (ILL), Grenoble. ${ }^{53}$ The reflectivity $R$ of the samples was determined as a function of the momentum change, $q$, perpendicular to the surface, where $q=4 \pi \sin \theta / \lambda$, and $\theta$ is the angle of incidence of the beam from the surface plane. Measurements were made with $\theta-2 \theta$ scans covering $q$-ranges from 0.01 to $0.25 \AA^{-1}$. Specularly reflected neutrons at $2 \theta$ were counted on a point detector, and the incident neutron flux on the sample was monitored for normalisation of the data. Beam collimation is controlled via two upstream slits, though for low $\theta$ (or $q$ ) the relatively large distance between the second slit and the sample reduces the neutron flux to unacceptable levels unless the sample is overilluminated. This was accounted for during data reduction, but it also increases the incoherent background noise. The background was checked $1^{\circ}$ off

the specular direction for some angles $\theta$, and was typically $5 \times 10^{-6}$ for the measurements in humid air (and with little $\theta$ dependence). Incident neutrons were reflected from the air side 
of the interface, and absolute reflectivities were obtained by normalizing the reflectivity to the totally reflected portion of the beam.

The specular reflectivity is determined by the scattering length density (SLD) profile, $\rho(z)$, perpendicular to the interface, and the very different scattering length densities of $\mathrm{D}_{2} \mathrm{O}$ and the protonated polymer were used to extract both thickness and water content from the reflectivity measurements.

The chamber for humidity control is made from aluminium and has two separate compartments connected via a slit, see Figure 1. The lower compartment is filled with $\mathrm{D}_{2} \mathrm{O}$, and the two compartments are temperature-controlled via separate circulator water baths. This arrangement permits the relative humidity to be varied continuously, and almost independently of the sample temperature. The sample temperature was set to $25^{\circ}$, and the humidity varied from $8 \%$ (obtained by flushing the chamber with $\mathrm{N}_{2}$ gas) to $98 \% \mathrm{RH}$ by varying the temperature of the lower compartment, with the $\mathrm{D}_{2} \mathrm{O}$ reservoir. The $\mathrm{RH}$ was monitored with a capacitive humidity sensor (Honeywell HIH-3610, pre-calibrated) connected to a voltmeter. After the measurements at the highest RH for each sample, the chamber was opened and the sample checked for condensation, though this was never observed.

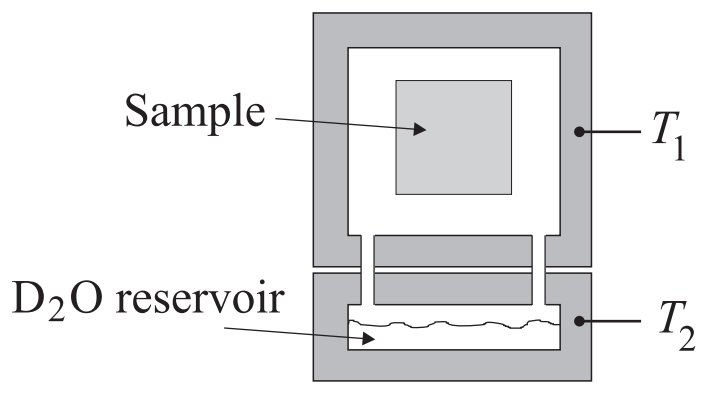

Figure 1: The chamber for control of relative humidity over the sample during the neutron reflectivity experiments. $T_{1}$ controls the sample temperature, and $T_{2}$ the relative humidity.

Data was fitted using multilayer slab models with interfacial roughness, using the Parratt32 program (HMI, Berlin). The interfacial roughness parameter $\sigma$ is the width of a Gaussian distribution between two layers. The goodness-of-fit was estimated by a $\chi^{2}$ parameter which is obtained by summing the squares of the residuals of the calculated reflectivity 
profiles. To reduce the number of parameters in the fitting of the hydrogel data, separate measurements were made on each block before hydrogel grafting, to determine the thicknesses and scattering length densities of the substrate layers.

The reflectivity from an interface in the kinematic approximation is given by

$$
R=\frac{16 \pi^{2}}{q^{4}}\left|\rho_{1}-\rho_{2}\right|^{2}
$$

where $\rho_{1}$ and $\rho_{2}$ are the scattering length densities on either side of the interface. The preferred substrate for the hydrogels in these experiments would be silicon because of the simple substrate structure, but as was mentioned above, a comparison of hydrogels on silica and gold is of interest for potential applications, and we also note that this may have the added advantage of improving the measurements on the hydrogel samples since the large SLD of the gold layer $\left(4.5 \times 10^{-6} \AA^{-2}\right)$ increases the total reflectivity from the interface.

\section{Results and discussion}

\section{FTIR-ATR measurements}

Infrared spectra were acquired on both sample types, and the $\mathrm{C}-\mathrm{H}$-stretching and fingerprint regions from spectra for both sample types are shown in Figure 2. The main features of the spectra are indicated in the figure, these are in agreement with previously published results for similarly prepared HEMA-co-PEG ${ }_{10}$ MA copolymers, see Larsson et al., ${ }^{25}$ which also includes a complete peak assignment. The spectra for the two sample types are very similar, with slight deviations in the $\mathrm{C}-\mathrm{H}$-stretching region, and a non-negligible deviation between the two spectra at $1110 \mathrm{~cm}^{-1}$ (indicated with a vertical line in the figure). The latter difference is caused by a negative contribution from the native $\mathrm{SiO}_{2}$ layer on the silicon substrate. For the spectrum of the polymer on gold, a clean gold substrate was used to obtain a background spectrum, and for the polymer on silicon, a clean piece of silicon 


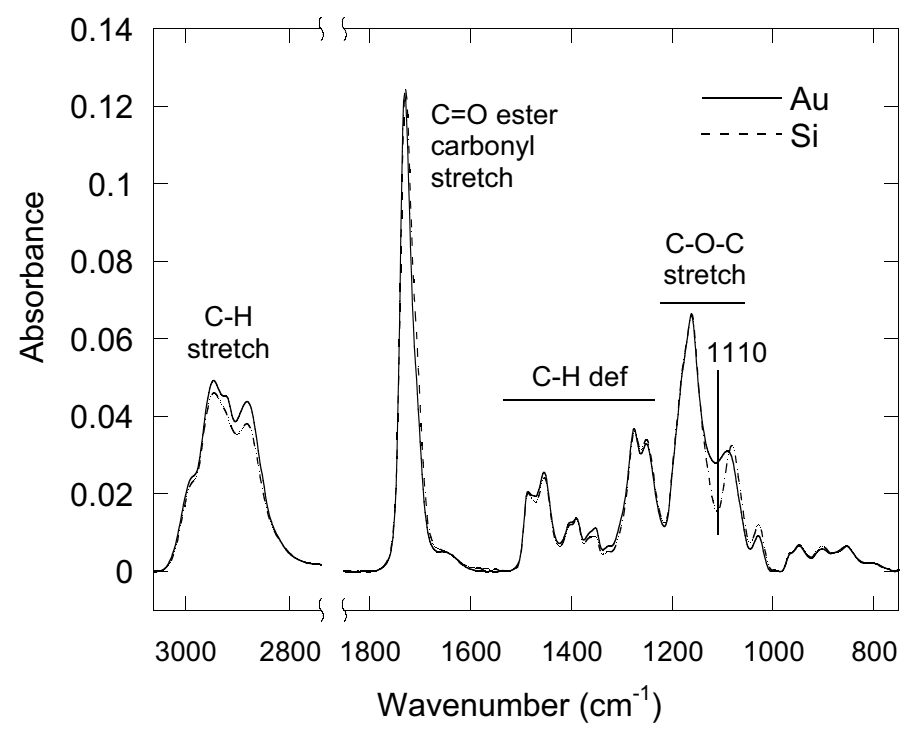

Figure 2: FTIR-ATR spectra of the thin HEMA-co-PEG ${ }_{10} \mathrm{MA}$ hydrogels prepared on $\mathrm{Au}$ and Si substrates, respectively.

was used for the background. However, the asymmetric Si-O-Si stretching vibration at $1110 \mathrm{~cm}^{-1}$ is very strong and would also be present in the background spectrum, but the contribution to this band is weaker from the hydrogel-coated sample than from the clean $\mathrm{SiO}_{2}$ reference, due to the thickness of the hydrogel layer bringing the $\mathrm{SiO}_{2}$ farther from the ATR prism. This reduction in absorbance emerges as a negative contribution to the spectrum. Hence, the reduced intensity for the sample on the silicon substrate near this particular wavenumber does not reflect a difference between the two polymer samples, and we conclude from the data that the polymers prepared on the two different substrate types are chemically very similar.

\section{Wettability}

The wettabilities of the hydrogels were investigated by recording advancing and receding contact angles at relative humidities between $0 \%$ and $\sim 95 \%$, see Figure 3 . Although there is some scatter in the data, the result of these measurements still indicate that there is no systematic change in either advancing or receding contact angle with $\mathrm{RH}$, and that the 


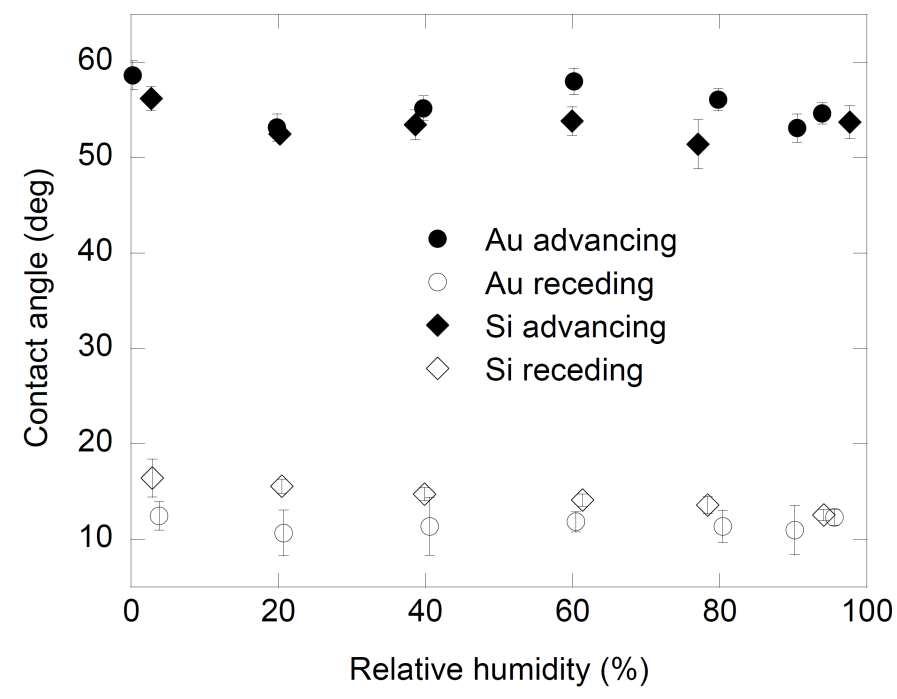

Figure 3: Advancing (filled symbols) and receding (empty symbols) contact angles on thin HEMA-co-PEG ${ }_{10} \mathrm{MA}$ films grafted from gold $(\mathrm{Au})$ and silicon (Si) substrates. Error bars represent standard deviations.

results are similar for the two sample types. There is also no measurable equilibration time; for $\sim 0 \%$ and $50 \%$ RH, no systematic differences were observed between contact angles measured immediately upon reaching a given humidity (ca two minutes waiting is required to stabilize the reading at a given $\mathrm{RH}$ value), and contact angles measured after overnight equilibration at these $\mathrm{RH}$ values.

The absence of variation in the advancing or receding contact angles in Figure 3 indicate that contact angle measurements are useful for characterization of these polymer films under ambient conditions, since the data is insensitive to humidity variations.

\section{Neutron reflectometry in humid $\left(\mathrm{D}_{2} \mathrm{O}\right)$ air}

Experiments in air of varying $\mathrm{D}_{2} \mathrm{O}$ humidity were performed with hydrogels grafted from both silica and gold substrates. Once a humidity reading was stable, no noticeable changes in hydrated polymer thicknesses were observed over the timescale of an experiment (counting for ca $5 \mathrm{~h}$ /humidity). Equilibration times $\leq 10 \mathrm{~s}$ have been reported for similar hydrogels, ${ }^{35}$ why equilibration of the hydrogel per se does not seem to be a problem. Reducing the humidity 
from $98 \%$ RH to approx. $5 \%$ by purging with dry nitrogen and cooling the $\mathrm{D}_{2} \mathrm{O}$ reservoir, could be accomplished within about one minute, and a control experiment conducted as soon as $\mathrm{RH}$ had reached $<5 \%$ showed that swelling was reversible, and did not show any signs of lag in swelling on this timescale.

Results for the hydrogel films grown on the silane-modified silicon substrates are shown in Figure 4a. The properties of the $\mathrm{Si} / \mathrm{SiO}_{2}$ substrate were determined in a separate experiment before grafting the hydrogel layer (during fitting, the $\mathrm{SiO}_{2}$ layer was kept constant at 13.4 $\AA$, with an SLD of $3.28 \times 10^{-6} \AA^{-2}$, accounting for some porosity of this layer, and the $\mathrm{SiO}_{2} /$ polymer interfacial roughness $6 \AA$ ). No model layer to account for the thin silane coupler anchoring the polymer to the surface is included in the models; including such a layer did not improve any fits. For humidities up to $80 \%$, the hydrated hydrogel can be modelled as a single slab with interfacial roughness, and additional layers either do not improve the fits, or converge to zero thickness during fitting. The fit parameters for the layers are summarized in Table 1. At 92\%, an additional layer of increased polymer density near the substrate is required to fit the data; Figure 5a shows the resulting SLD profiles. To fit the data at $98 \%$ $\mathrm{RH}$, a similar layer of increased polymer density near the substrate is needed, but also an additional layer of decreased SLD near the air interface, indicating accumulation of polymer chains near this surface. Further details about the fitting are provided in the Supporting Information, this includes a discussion on the deviations between the data and the fits at low $q$ for the lowest humidities, and also a potential source of error for $98 \% \mathrm{RH}$, and the results of using one- and two-layer models for the $\mathrm{RH} 98 \%$ data.

Taking the density of the hydrogel to be that of poly(HEMA), i.e. $1.15 \mathrm{~g} / \mathrm{ml}$ (Sigma), and assuming that the HEMA:PEG ${ }_{10} \mathrm{MA}$ ratio is $1: 1$, the SLD of the polymer will be $\rho_{p}=$ $0.81 \times 10^{-6} \AA^{-2}$, and together with the SLD of $\mathrm{D}_{2} \mathrm{O}, \rho_{w}=6.35 \times 10^{-6} \AA^{-2}$, the water content (by volume) $\phi_{w}$ in the hydrogel can be calculated from $\rho_{w}$ and the measured SLD, 

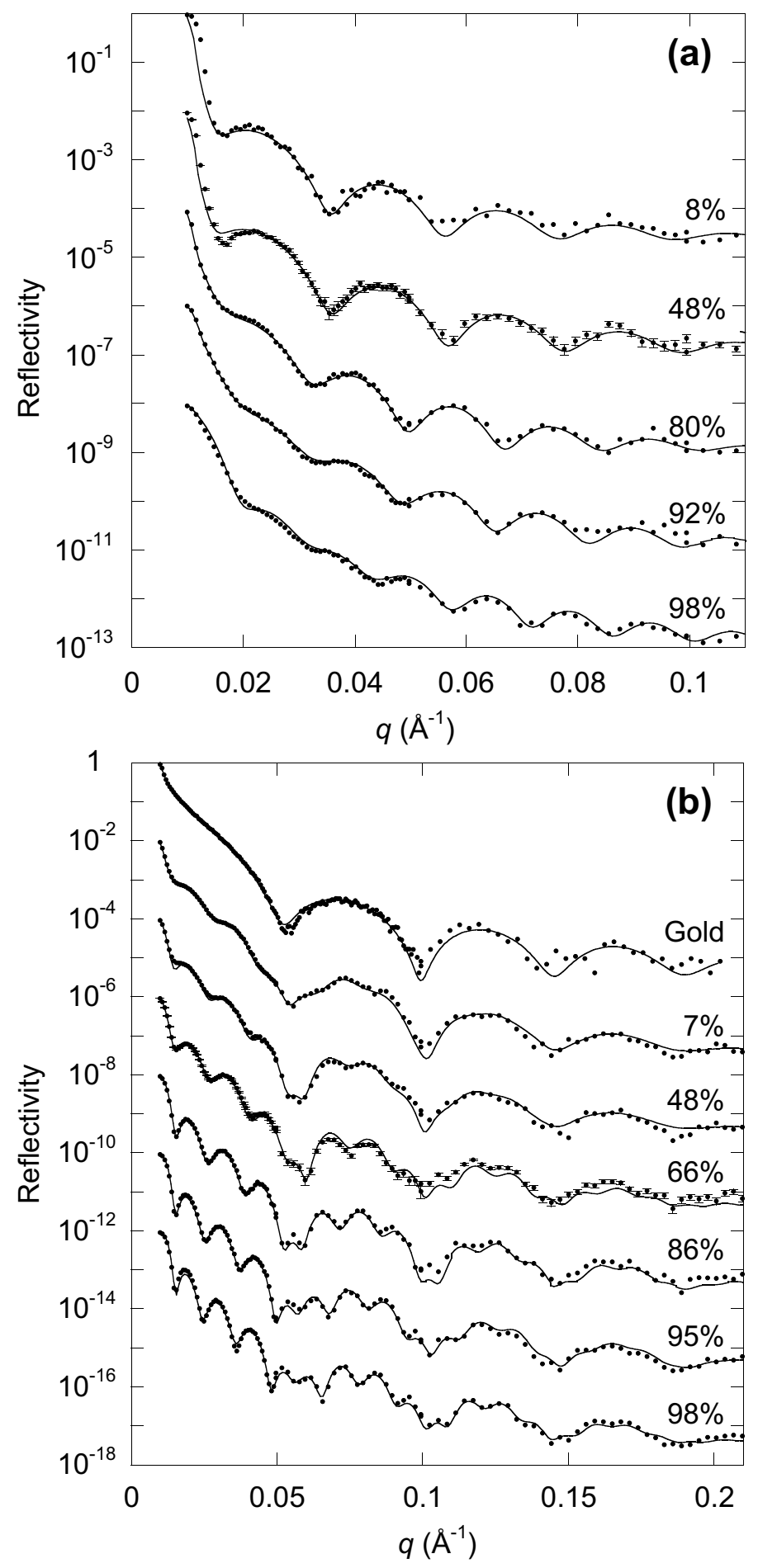

Figure 4: Reflectivities for hydrogels on (a) silicon (b) and gold substrates in atmospheres of different relative humidity. The first data set in (b) is for the clean gold substrate. Experimental data are shown as points, and the solid lines are fits to the data. Each data set is scaled by a factor of 0.01 relative to the preceding, and error bars are shown only on one data set in each graph, for clarity; the errors are of similar magnitude for all data sets. Note the different $q$ ranges in (a) and (b). 
Table 1: Fit parameters and calculated properties for hydrogels grafted from silicon. The data at $92 \%$ and $98 \% \mathrm{RH}$ are given with the layers near the substrate first.

\begin{tabular}{|c|c|c|c|c|c|c|c|}
\hline \multirow[b]{2}{*}{$\begin{array}{l}\text { RH } \\
(\%)\end{array}$} & \multicolumn{3}{|c|}{ Hydrogel } & \multirow{2}{*}{$\begin{array}{c}\text { Water } \\
\text { content } \\
(\%)\end{array}$} & \multirow{2}{*}{$\begin{array}{c}\text { Thickness } \\
\text { increase }^{1} \\
(\%)\end{array}$} & \multirow{2}{*}{$\begin{array}{c}\text { Equivalent } \\
\text { dry thickness } \\
(\AA)\end{array}$} & \multirow[b]{2}{*}{$\chi^{2}$} \\
\hline & $\begin{array}{c}d \\
(\AA)\end{array}$ & $\begin{array}{c}\text { SLD } \\
\left(\times 10^{-6} \AA^{-2}\right)\end{array}$ & $\begin{array}{c}\sigma \\
(\AA)\end{array}$ & & & & \\
\hline 8 & 278 & 1.42 & 12 & 11.0 & 18.4 & 247 & 0.0981 \\
\hline 48 & 295 & 1.57 & 15 & 13.7 & 22.1 & 255 & 0.0907 \\
\hline 80 & 349 & 1.96 & 12 & 20.7 & 43.0 & 277 & 0.0448 \\
\hline \multirow[t]{2}{*}{92} & 70 & 2.20 & 30 & & & & \\
\hline & 295 & 2.54 & 8 & 28.3 & 48.8 & 265 & 0.0377 \\
\hline \multirow[t]{3}{*}{98} & 57 & 2.41 & 28 & & & & \\
\hline & 306 & 3.04 & 11 & 34.3 & 72.1 & 273 & 0.0276 \\
\hline & 58 & 2.89 & 8 & & & & \\
\hline
\end{tabular}

${ }^{1}$ Relative to the dry thickness extrapolated from RH $8 \%$.
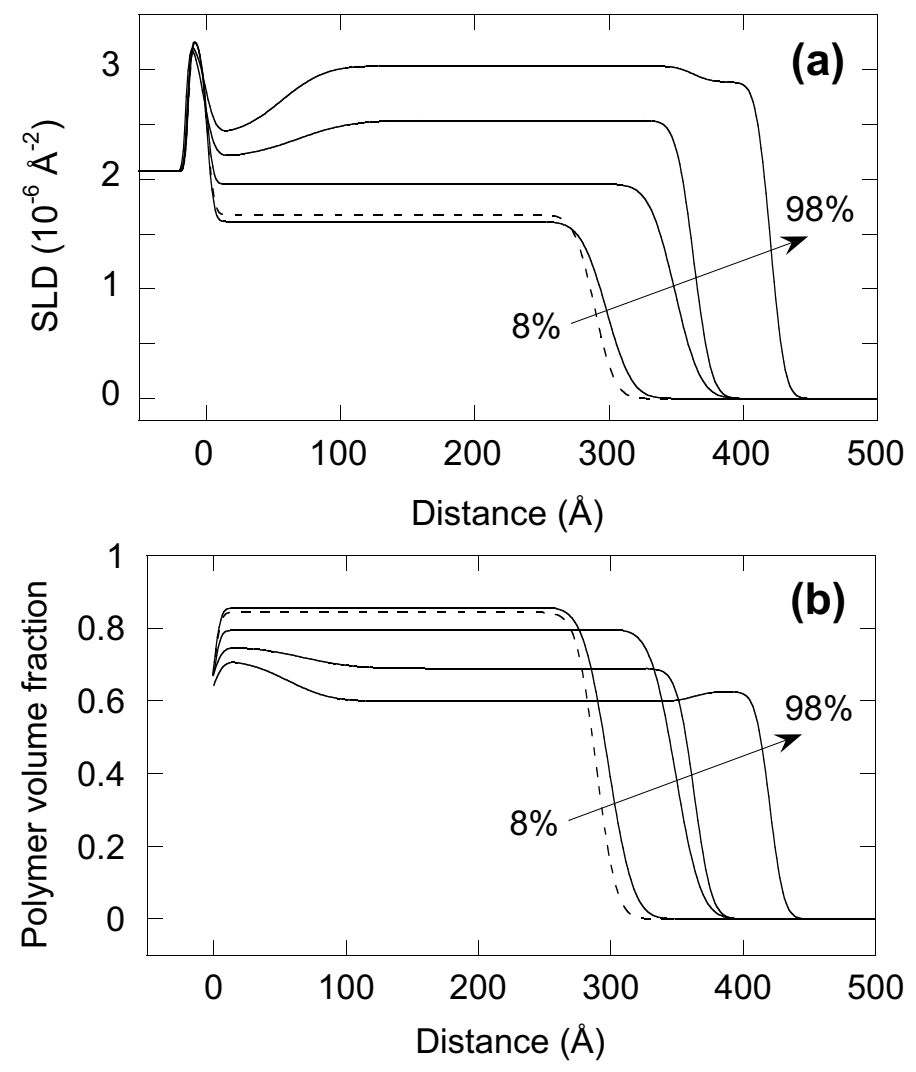

Figure 5: (a) The SLD profiles for the HEMA-co-PEG ${ }_{10}$ MA hydrogel on Si substrates, and (b) the resulting polymer chain segment density profiles, as calculated from the SLD profiles in (a). Zero distance is taken at the $\mathrm{SiO}_{2} /$ polymer interface, and the slight increase seen there for all polymer density profiles in (b) is a result of the assigned roughness of this interface. 
$\rho_{\text {exp }}$, at each humidity, under the assumption that the volumes are additive:

$$
\rho_{e x p}=\rho_{w} \phi_{w}+\rho_{p}\left(1-\phi_{w}\right) \Rightarrow \phi_{w}=\frac{\rho_{e x p}-\rho_{p}}{\rho_{w}-\rho_{p}}
$$

With only two components in the system, the polymer volume fraction $\phi_{p}=1-\phi_{w}$ is obtained, and are shown in Figure 5b for all humidities. Calculated values of the total water content and the swelling for all humidities are included in Table 1 (for completeness, figures explicitly showing the water volume fractions $\phi_{w}$ are included in the Supporting Information).

Results from the samples on gold are shown in Figure 4b. Characterization of the goldcoated block before grafting the hydrogel resulted in the parameters in Table 2. These were kept fixed as the reflectivity profiles from the measurements with the hydrogel were subsequently analysed.

A single slab with interfacial roughness was sufficient to model the polymer layer at all humidities; additional layers near the substrate or at the air interface, like those required for the $92 \%$ or $98 \%$ RH data on silicon, were not necessary (i.e., did not improve the fits, or converged to zero thickness). The resulting reflectivity profiles are included in Figure 4b, and the obtained parameters are summarized in Table 3, with SLD profiles and the resulting polymer chain segment density distributions shown in Figure 6 (distributions for water are included in Supporting Information). Note that the $\chi^{2}$-values in Tables 1 and 3 cannot be compared between the tables, since the data sets contain different numbers of points and cover different $q$-ranges, and are only meaningful for comparisons between data sets within each table.

Although the thickness of the alkylthiol layer is less than $5 \%$ of the hydrogel thickness, its relative impermeability to water gives it an SLD which is quite different from that of the hydrogel, and as such it needs to be carefully taken into account in the models. The thickness of this layer is constant for all humidities, though the water content increases slightly with the RH, as can be seen from the slowly increasing SLD for this layer; the SLD 
Table 2: Properties of the gold substrate

\begin{tabular}{cccc}
\hline & $d$ & SLD & $\sigma$ \\
Layer & $(\AA)$ & $\left(\times 10^{-6} \AA^{-2}\right)$ & $(\AA)$ \\
\hline $\mathrm{Au}$ & 137 & 4.50 & 9 \\
$\mathrm{Ti}$ & 9.64 & -1.96 & 5 \\
$\mathrm{SiO} 2$ & 10.3 & 3.39 & 5 \\
$\mathrm{Si}$ & $\infty$ & 2.07 & 3 \\
\hline
\end{tabular}

Table 3: Fit parameters and calculated properties for hydrogels grafted onto gold.

\begin{tabular}{|c|c|c|c|c|c|c|c|c|c|c|}
\hline \multirow[b]{2}{*}{$\begin{array}{l}\mathrm{RH} \\
(\%)\end{array}$} & \multicolumn{3}{|c|}{ Alkylthiol layer } & \multicolumn{3}{|c|}{ Hydrogel } & \multirow{2}{*}{$\begin{array}{c}\text { Water } \\
\text { content } \\
(\%)\end{array}$} & \multirow{2}{*}{$\begin{array}{c}\text { Thickness } \\
\text { increase }^{1} \\
(\%)\end{array}$} & \multirow{2}{*}{$\begin{array}{c}\text { Equivalent } \\
\text { dry thickness } \\
(\AA)\end{array}$} & \multirow[b]{2}{*}{$\chi^{2}$} \\
\hline & $\begin{array}{l}d \\
(\AA)\end{array}$ & $\begin{array}{c}\text { SLD } \\
\left(\times 10^{-6} \AA^{-2}\right)\end{array}$ & $\begin{array}{c}\sigma \\
(\AA)\end{array}$ & $\begin{array}{l}d \\
(\AA)\end{array}$ & $\begin{array}{c}\text { SLD } \\
\left(\times 10^{-6} \AA^{-2}\right)\end{array}$ & $\begin{array}{c}\sigma \\
(\AA)\end{array}$ & & & & \\
\hline 7 & 18.4 & -0.37 & 10 & 329 & 1.16 & 16 & 6.3 & 4.5 & 308 & 0.0441 \\
\hline 48 & 18.3 & -0.02 & 12 & 357 & 1.73 & 15 & 16.6 & 16.6 & 298 & 0.0781 \\
\hline 66 & 17.8 & 0.14 & 11 & 360 & 1.85 & 15 & 18.8 & 16.9 & 293 & 0.0903 \\
\hline 86 & 17.5 & 0.15 & 12 & 379 & 2.28 & 8 & 26.5 & 23.1 & 279 & 0.0891 \\
\hline 95 & 17.9 & 0.46 & 10 & 405 & 2.39 & 9 & 28.5 & 31.5 & 290 & 0.0726 \\
\hline 98 & 17.6 & 0.51 & 12 & 424 & 2.65 & 7 & 33.2 & 37.7 & 283 & 0.0896 \\
\hline
\end{tabular}

${ }^{1}$ Relative to the dry thickness extrapolated from $\mathrm{RH} 7 \%$.

of a pure hydrocarbon is $-0.5 \times 10^{-6} \AA^{-2}$. Thiohexadecanol monolayers on gold form dense, crystalline monolayers after overnight incubation, but it is possible that the UV irradiation during the polymerization somewhat damages the layer, and thus permits penetration of up to $15 \%$ water at the highest $\mathrm{RH}$.

The differences in UV exposure time needed to prepare the hydrogels on gold and silicon (3 and 10 minutes, respectively) are not quantitatively reflected in the thickness difference between the films, in agreement with the empirical observation that the growth of the films proceeds differently on these substrates, and that even a greater amount of polymer is grafted onto the 3-minute exposed gold substrate, than on the 10-minute exposed silica substrate. The average dry thicknesses on the silicon and gold substrates are $263 \AA$ and $292 \AA$, as obtained by averaging the rightmost columns in Tables 1 and 3, respectively. Considering the very different growth rates, there are likely also differences in the internal structure of these films that affect the swelling. (Differences in UV reflectivity cannot explain the faster growth on gold than on silicon; the reflectivity from silicon is higher than that for gold 

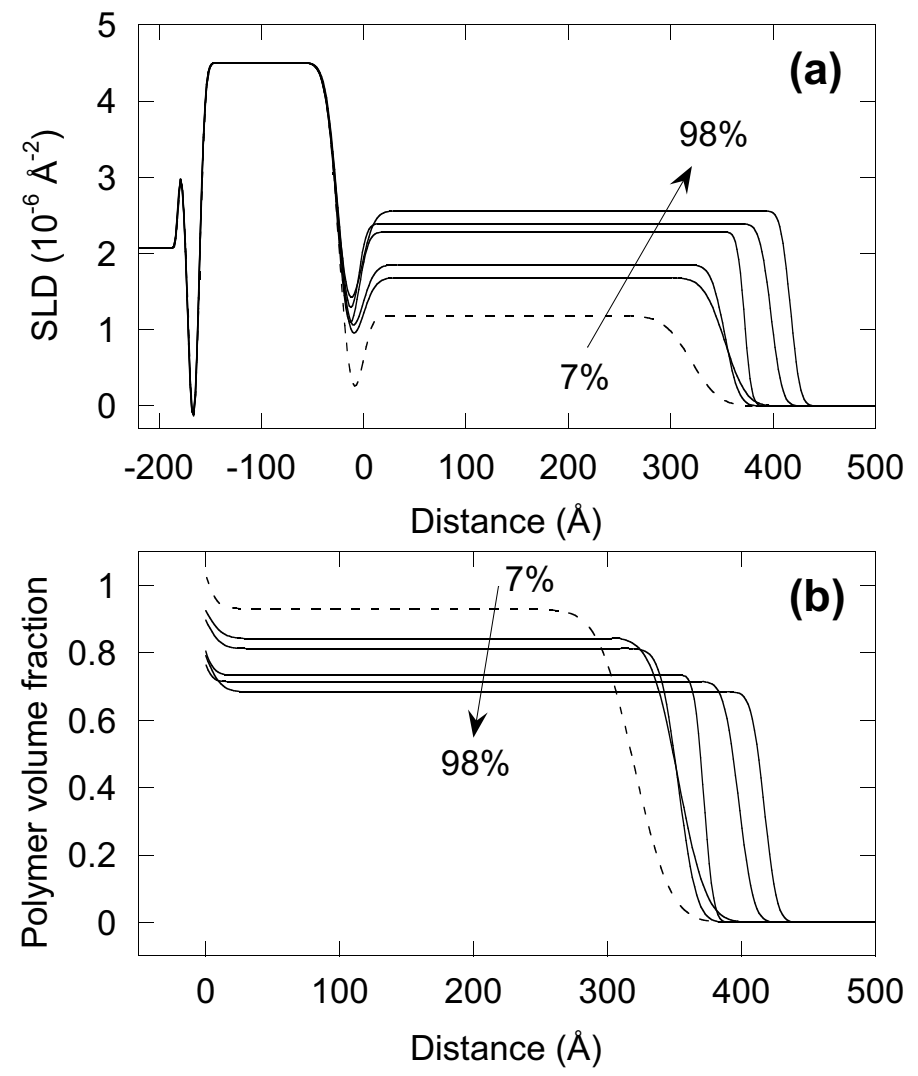

Figure 6: (a) The SLD profiles for the hydrogel on Au substrates, and (b) the resulting polymer density profiles, as calculated from the SLD profiles in (a). Zero distance is taken at the alkylthiol/polymer interface, and the slight decrease seen there for all polymer density profiles in (b) is a result of the assigned constant roughness of this interface. 
throughout the relevant part of the UV spectrum, as is shown in the Supporting Information.)

The polymer density profiles on the silicon substrate (Figure 5), and the constant profiles obtained for the films on gold (Figure 6), are both qualitatively different from segment density profiles observed in, for example, polyelectrolyte brushes hydrated by water vapour, ${ }^{50}$ where depletion of the polymer at both interfaces was observed, rather than the accumulation at both interfaces, as is shown for the highest humidities in Figure 5, though the effects are also much less pronounced in our case. Comparisons with brushes swollen in (bulk) water are less informative, due to the absence of a free interface to air, whose surface tension is a constraint to the swelling in vapours. However, we note that oligo(ethylene glycol) methyl ether methacrylate (OEGMA) brushes swollen in water show a continuous chain segment density decrease with distance from the substrate, ${ }^{41}$ and similar results were obtained for HEMA co-polymerized with methacrylic $\operatorname{acid}^{54}$ and poly(2-(dimethylamino)ethyl methacrylate)) brushes. ${ }^{55}$ These differences indicate that the structure of the SIPGP films under consideration here, differ in significant ways from regular polymer brushes, giving support to the hypothesis that the UV-induced polymerization could result in cross-linking of the layers, and formation of a bush-like polymer, as a result of the unspecific free radical formation under UV illumination. ${ }^{56}$

To ensure that the observed differences in the thickness changes are not the result of inappropriate fitting of the data, the equivalent dry polymer thickness was calculated for all entries in Tables 1 and 3 (by subtracting the water volume fraction from the total thickness). If properly done, this should be a constant for each sample, independent of the RH, and the actual variation is within $\pm 6 \%$ for the silicon samples, and $\pm 5 \%$ for the gold samples, These variations are significantly less than the thickness changes shown in Figure 7 . This also demonstrates that the observed difference in swelling is not the result of polymer chains "dangling" outside the $\mathrm{D}_{2} \mathrm{O}$ layer, and thus being invisible to the neutrons due to the low contrast between the polymer and the surrounding air. It is also important to emphasize that the difference in relative increase of the two layer thicknesses is not a result of uncertainties in 
the fitting of the data in Figure 4. The Kiessig fringes accurately provide the thicknesses of the polymer layers, and additional layers (as used for RH $92 \%$ and $98 \%$ on silicon) primarily improve the fits by allowing a local deviation of the SLD, but do not significantly alter the total polymer thickness, as compared to a best fit with a one-layer model. The variations in total thickness for one-, two- and three-layer models for the $98 \% \mathrm{RH}$ on silicon is $<4 \%$ (see the Supporting Information). However, the difference in swelling is obvious already at lower $\mathrm{RH}$ where the fits to one-layer models are excellent.

While the results summarized in Tables 1 and 3, and in Figure 7, point at clear differences, there are also some similarities between the thin hydrogel films on the two substrates. In both cases, the hydrogels can be modelled as layers with homogeneous composition and moderate roughness at low humidities. This shows that the water is evenly distributed throughout the film, and in particular that there is no water-depleted layer in the polymer. As the RH is increased, the hydrogels are swelling, and both the thicknesses of the hydrogels and the SLDs of the layers increase, reflecting the uptake of $\mathrm{D}_{2} \mathrm{O}$ into the film. For the most swollen films, however, there are clear qualitative differences between the two film types, in addition to the obvious differences in swelling.

The variations in thickness and SLD with RH are summarized in Figure 7, from which it is clear that the change in thickness is steeper for humidities near saturation, and thus that variations in humidity will have a greater impact on the thickness at higher RH. Figure 7 also reveals a striking difference between the swelling of the two hydrogel films, with considerably greater swelling of the film formed on $\mathrm{Si}$, compared to that on $\mathrm{Au} ; 72 \%$ at $98 \% \mathrm{RH}$ on $\mathrm{Si}$, compared to $38 \%$ at $98 \% \mathrm{RH}$ on $\mathrm{Au}$ (as was mentioned before, no visible condensation of water could be seen after the experiments at the highest humidities). Still, the swelling profiles are in qualitative agreement with observations on pHEMA under similar conditions. $^{57}$

It is interesting that the variations in SLD for the two films are very similar over the range of humidities (Figure 7), since this implies that the water:polymer ratio is approximately in- 


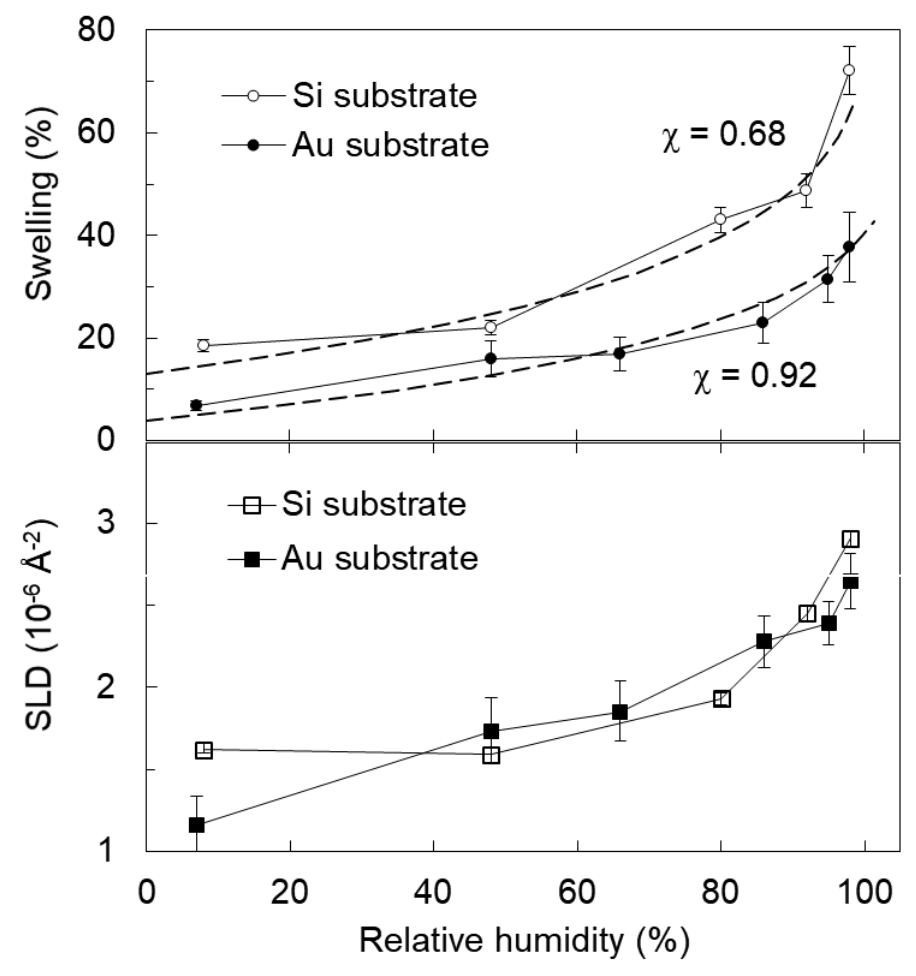

Figure 7: Changes in thickness ( $d$, circles) and scattering length density (SLD, squares) for hydrogels on gold (Au, filled symbols) and silicon ( $\mathrm{Si}$, open symbols), versus the relative humidity (RH). The error bars for the swelling values were obtained by varying the thickness of the polymer layer (for $92 \%$ and $98 \% \mathrm{RH}$ on silicon, the thickness of the thickest polymer layer) and using the thicknesses that resulted in a $5 \%$ increase in $\chi^{2}$. The error bars for the SLD values similarly represent the deviations required to cause a $5 \%$ increase in $\chi^{2}$. The consistently larger errors found on the Au substrate reflect the fact that these fits are less sensitive to changes in the polymer properties in the model, since the reflectivity is dominated by the contribution from the gold film. The SLD errors for silicon are smaller than the symbols in the plot. The top pane also shows the results of fitting a Flory-Hugginstype sorption model to the data, with the resulting interaction parameters displayed; see text for details. 
dependent of the differences in swelling, and also independent of any possible structural differences (see below). As the RH approaches 100\%, the thickness of both samples increase faster than the SLD (the amount of imbibed water), indicating a volume expansion of the PEG chains as the increasing water content increases their solubility. The swelling of the polymer films upon increasing $\mathrm{RH}$ is considerably greater for the film grown on the silicon substrate, suggesting a larger polymer chain segment volume at a given RH. It is conceivable that since the polymerization proceeds at different rates on the two substrates, the structure of the polymers are different, for example in the average chain lengths, or in the degree of cross-linking, and that this affects the volume expansion upon hydration. The decreasing surface roughness $\sigma$ of the hydrogel/air interfaces upon increasing $\mathrm{RH}$ is significant for the hydrogels on both substrates, as seen in Tables 1 and 3, and is probably related to the increasing importance of surface tension as the amount of water increases, and the hydrogel/air interface becomes more fluid.

The polymer-solvent interaction (or Flory-Huggins) parameter, $\chi$, is a measure of the strength of interaction between a polymer and its solvent, and is predicted by Flory-Huggins theory to be a constant, independent of volume fraction for a given polymer and temperature, ${ }^{58}$ and studies show that it is near 0.43 for PEG hydrogels over a wide range of volume fractions. ${ }^{59}$ However, as discussed by Akalp et al. recently, ${ }^{60}$ other studies indicate that this is an oversimplicifation, and that $\chi$ varies with both volume fraction ${ }^{61}$ and molecular weight, ${ }^{62}$ and a generalized Flory-Huggins theory has been shown even to semiquantitatively explain experimental temperature-concentration and temperature-pressure phase diagrams of PEG solutions. ${ }^{63}$ For a hydrogel, $\chi$ is also affected by the degree of cross-linking, and how water is associated with the polymer. ${ }^{64}$ Hence, it is to be expected that the polymer-solvent interaction parameter for PEG-containing hydrogels in water will vary with the polymer chemistry, and thus also with processing conditions, as well as with the details of the composition, cross-linking and possible degradation.

The swelling in the studied hydrogel films can be modelled as a Flory-Huggins-type sorp- 
tion behaviour, as described by Biesalski and Rühe. ${ }^{65}$ Here, water is a permeant which interacts more strongly with itself than with the polymer, leading to an exponentially increasing absorption with pressure, described by the Flory-Huggins relation

$$
\ln \frac{p}{p_{0}}=\ln \phi_{w}+\left(1-\phi_{w}\right)+\chi\left(1-\phi_{w}\right)^{2}
$$

where $p$ is the vapour pressure, $p_{0}$ the saturation vapour pressure, and $\chi$ the Flory-Huggins interaction parameter. For the moderate swelling observed here, we assume that free energy changes due to chain stretching within the polymer are small. Equation 2 was fitted to the swelling data in Figure 7, to obtain the Flory-Huggins interaction parameters for the two polymers; $\chi_{\mathrm{Si}}=0.68$ and $\chi_{\mathrm{Au}}=0.92$. The fits, shown in Figure 7 , allow for residual water in the hydrogels when extrapolated to $0 \% \mathrm{RH}$ (13\% for Si and $4 \%$ for $\mathrm{Au})$. A higher value of the interaction parameter implies a less favourable polymer-solvent interaction. The discrepancy between $\chi_{\mathrm{Si}}$ and $\chi_{\mathrm{Au}}$ is an indication that there are differences in the way water interacts with the polymers on the two different substrates. Notably, these two values are greater than the value $\chi=0.43$ for regular PEG hydrogels, ${ }^{59}$ and are also greater than what is observed for PEG in solution. Pedersen et al. ${ }^{66}$ found that $\chi$ for PEG in water varies from 0.32 to 0.52 in the temperature range $10-100{ }^{\circ} \mathrm{C}$. Both $\chi_{\mathrm{Si}}$ and $\chi_{\mathrm{Au}}$ are thus greater than $\chi$ for either a PEG hydrogel or for free PEG in solution, even at high temperatures when water is a poor solvent for PEG. The difference between $\chi_{\mathrm{Si}}$ and $\chi_{\mathrm{Au}}$ would not be possible without some distinct differences between the hydrogels on the two substrates. The swelling capacity of a hydrogel is governed primarily by the chemical structure, molecular weight and the crosslinking ratio (in addition to parameters that do not vary between our two systems or do not apply in this case, such as solvent quality and concentration, interpenetrating network structure, specific stimuli, or surrounding medium). The types and starting compositions of the monomers are identical in the two cases, and while differences in resulting monomer ratios in the films are possible in principle, there is no evidence for such chemical inhomogeneities. 
Instead, we propose to look for differences in the structure caused directly or indirectly by the UV-polymerization process. In the previous, differences in reflectivity of the substrates were ruled out as a possible explanation for the faster growth on the gold surface. Another possibility is that quenching of the UV-generated free radicals has some importance, though we have not been able to find support for such an explanation in the literature, or reports relevant to $\mathrm{Si}$ or Au substrates in this respect.

A remaining possible origin of the differences in swelling and polymer interaction parameter, and also of the enrichment of polymer near the interfaces for the silicon substrate that is not observed on gold, is the prolonged UV exposure required to grow the polymer on silicon, causing increased damage, and possibly also cross-linking of the polymer. Radiation processing of polymers such as PEO or polyethylene with ionizing radiation is widely used, resulting in crosslinking and chain scissioning, as a result of formation of hydroxyl and hydrogen radicals in the presence of water. This is also the effect of UV irradiation. The polymer near the substrate surface is exposed by the damaging radiation for longer, and hence is more severely affected. On gold, the polymer grows faster, resulting in a relatively homogeneous film, that is, a more brush-like structure, that does not permit as much extension of the polymer chains upon hydration. On the other hand, if the much longer UV-exposure of the polymer formed on silicon causes a more heterogeneous structure, with possible cross-linking forming a dense layer near the surface, and greater variation in chain lengths, this structure might allow for larger swelling, and also accumulation of the longest chain ends near the liquid/air interface, giving rise to the slight increase in polymer density at the interface to air, obtained on the silicon-supported films. There is some support to this view in the IR data in Figure 2; the decrease in intensity in the $\mathrm{C}-\mathrm{H}$-region for the sample on silicon could indicate that $\mathrm{C}-\mathrm{H}-$ bonds are present to a lesser extent. Cross-linking would replace $\mathrm{C}-\mathrm{H}$-bonds with $\mathrm{C}-\mathrm{C}$-bonds. The latter are weak in the infrared, but could possibly explain a slight increase in intensity of the two peaks between 1100 and $1000 \mathrm{~cm}^{-1}$ for the sample on silicon. 
Degradation or damage caused by the UV illumination is a known negative effect of the SIPGP UV-polymerization procedure ${ }^{25}$ but in contrast to more well-controlled methods, such as atom transfer radical polymerization (ATRP) ${ }^{67}$ or reversible addition-fragmentation chain transfer (RAFT) polymerization, ${ }^{68}$ the SIPGP method allows for rapid and simple polymerization on virtually any organic substrate without the need for initiators, and allows for simple preparation of patterns ${ }^{27}$ or gradients, ${ }^{56}$ and can be applied to surfaces or materials

of very different geometries. ${ }^{69}$ Hence, these advantages of the SIPGP methods in certain contexts and applications, motivates continuing investigation into this process.

\section{Summary and conclusions}

We studied the swelling of thin UV-polymerized SIPGP hydrogel films grown on silica and gold substrates, monitoring thickness increase and water uptake at varying relative humidities. The polymer growth proceeds at different rates on the two types of substrate. Resulting films with similar thicknesses show clear differences in swelling with the type of substrate. This difference is attributed to structural differences emerging as a result of differences in UV-exposure during polymerization, and is most pronounced upon swelling of the polymers, where inhomogeneities in the chain segment density distribution is apparent for films grafted from the silicon substrate. This is supported by fitting the swelling data to a Flory-Hugginstype sorption model, yielding polymer interaction parameters $\chi$ which are distinctly different on the two substrate types. The observed polymer density distributions for both types are also distinctly different from those observed for polymer brushes, supporting previous suggestions that these UV-polymerized hydrogel films have some degree of cross-linking, a hypothesis that is weakly supported by the infrared study, showing that polymers on the two types of substrates have otherwise similar chemical structure. Wetting studies showed that both advancing and receding contact angles were independent of the surrounding humidity. This is a useful observation for characterization purposese, but stands in contrast to, for 
example, the water uptake of the films, which did not show any region where the thickness did not vary with the humidity, for any of the two types. The benefits of SIPGP-prepared polymers over more well-controlled methods (e.g. ATRP or RAFT) for certain purposes, motivates further studies of these polymer films. Our observations also have wider practical implications, since thin hydrogel films on both gold and silicon substrates are widely used in biosensing and antifouling applications, but clearly should not be assumed to be structurally identical.

\section{Acknowledgement}

We gratefully acknowledge ILL beamtime on the ADAM instrument (experiment 9-11-1290) and technical assistance from Max Wolff. We also thank Christopher Aronsson, Katarina Bengtsson and Henrik Höckerdal who designed an early version of the humidity cell for the wettability tests. This work has been supported by the European Commission's 6th Framework Programme via the AMBIO project (NMP-CT-2005-011827), and by the Swedish Research Council (Vetenskapsrådet, dnr 2014-4004).

\section{Supporting Information Available}

Details about the fitting of the reflectivity data and a comparison of 1-, 2- and 3-layer models. Water volume fraction profiles, and reflectivity data on silicon and gold in the UV.

The following files are available free of charge.

- Filename: Ederth-supp.pdf

\section{References}

(1) Laftah, W. A.; Hashim, S.; Ibrahim, A. N. Polymer Hydrogels: A Review. PolymerPlastics Technology and Engineering 2011, 50, 1475-1486. 
(2) Nonoyama, T.; Gong, J. P. Double-network hydrogel and its potential biomedical application: A review. Proc. Inst. Mech. Eng., Part H 2015, 229, 853-863.

(3) Utech, S.; Boccaccini, A. R. A review of hydrogel-based composites for biomedical applications: enhancement of hydrogel properties by addition of rigid inorganic fillers. J. Mater. Sci. 2016, 51, 271-310.

(4) Parlato, M.; Reichert, S.; Barney, N.; Murphy, W. L. Poly(ethylene glycol) Hydrogels with Adaptable Mechanical and Degradation Properties for Use in Biomedical Applications. Macromol. Biosci. 2014, 14, 687-698.

(5) Hotz, N.; Wilcke, L.; Weber, W. Design, Synthesis, and Application of Stimulus-Sensing Biohybrid Hydrogels. Macromol. Rapid Commun. 2013, 34, 1594-1610.

(6) Hoffman, A. S. Hydrogels for biomedical applications. Adv. Drug Delivery Rev. 2002, $54,3-12$.

(7) Caló, E.; Khutoryanskiy, V. V. Biomedical applications of hydrogels: A review of patents and commercial products. Eur. Polym. J. 2015, 65, 252-267.

(8) Faxälv, L.; Ekblad, T.; Liedberg, B.; Lindahl, T. L. Blood compatibility of photografted hydrogel coatings. Acta Biomater. 2010, 6, 2599-2608.

(9) Kapoor, S.; Kundu, S. C. Silk protein-based hydrogels: Promising advanced materials for biomedical applications. Acta Biomater. 2016, 31, 17-32.

(10) Costa, D.; Valente, A. J. M.; Miguel, M. G.; Queiroz, J. Plasmid DNA hydrogels for biomedical applications. Adv. Colloid Interface Sci. 2014, 205, 257-264.

(11) Dånmark, S.; Aronsson, C.; Aili, D. Tailoring Supramolecular Peptide-Poly(ethylene glycol) Hydrogels by Coiled Coil Self-Assembly and Self-Sorting. Biomacromolecules 2016, 17, 2260-2267. 
(12) Buenger, D.; Topuz, F.; Groll, J. Hydrogels in sensing applications. Prog. Polym. Sci. 2012, 37, 1678-1719.

(13) Mateescu, A.; Wang, Y.; Dostalek, J.; Jonas, U. Thin Hydrogel Films for Optical Biosensor Applications. Membranes 2012, 2, 40.

(14) Nishiyabu, R.; Ushikubo, S.; Kamiya, Y.; Kubo, Y. A boronate hydrogel film containing organized two-component dyes as a multicolor fluorescent sensor for heavy metal ions in water. J. Mater. Chem. A 2014, 2, 15846-15852.

(15) Julian, G.; Christoph, S.; Gerald, U. Application of hydrogel-coated microcantilevers as sensing elements for pH. J. Micromech. Microeng. 2009, 19, 127002.

(16) Park, J.; Hong, W.; Kim, C. S. Color Intensity Method for Hydrogel Oxygen Sensor Array. IEEE Sens. J. 2010, 10, 1855-1862.

(17) Tellis, J. C.; Strulson, C. A.; Myers, M. M.; Kneas, K. A. Relative Humidity Sensors Based on an Environment-Sensitive Fluorophore in Hydrogel Films. Anal. Chem. 2011, 83, 928-932.

(18) Pernice, R.; Adamo, G.; Stivala, S.; Parisi, A.; Busacca, A. C.; Spigolon, D.; Sabatino, M. A.; D’Acquisto, L.; Dispenza, C. Opals infiltrated with a stimuliresponsive hydrogel for ethanol vapor sensing. Opt. Mater. Express 2013, 3, 1820-1833.

(19) Topuz, F.; Buenger, D.; Tanaka, D.; Groll, J. In Comprehensive Biomaterials; Ducheyne, P., Ed.; Elsevier: Oxford, 2011; pp 491-517.

(20) Löfås, S.; Johnsson, B. J. A novel hydrogel matrix on gold surfaces in surface-plasmon resonance sensors for fast and efficient covalent immobilization of ligands. J. Chem. Soc. Chem. Commun. 1990, 21, 1526.

(21) Harris, J. M. Poly(Ethylene Glycol) Chemistry: Biotechnical and Biomedical Applications; Plenum Press: New York, 1992. 
(22) Elbert, D. L.; Hubbell, J. Surface treatments of polymers for biocompatibility. Annu. Rev. Mater. Sci. 1996, 26, 365-370.

(23) Zhang, F.; Kang, E. T.; Neoh, K. G.; Huang, W. Modification of gold surface by grafting of poly(ethylene glycol) for reduction in protein adsorption and platelet adhesion. $J$. Biomater. Sci., Polym. Ed. 2001, 12, 515-531.

(24) Roosjen, A.; de Vries, J.; van der Mei, H. C.; Norde, W.; Busscher, H. J. Stability and effectiveness against bacterial adhesion of poly(ethylene oxide) coatings in biological fluids. J. Biomed. Mater. Res., Part B 2005, 73B, 347-354.

(25) Larsson, A.; Ekblad, T.; Andersson, O.; Liedberg, B. Photografted Poly(ethylene glycol) Matrix for Affinity Interaction Studies. Biomacromolecules 2007, 8, 287-295.

(26) Zhu, J. Bioactive modification of poly(ethylene glycol) hydrogels for tissue engineering. Biomaterials 2010, 31, 4639-4656.

(27) Larsson, A.; Du, C.-X.; Liedberg, B. UV-Patterned Poly(ethylene glycol) Matrix for Microarray Applications. Biomacromolecules 2007, 8, 3511-3518.

(28) Ekblad, T.; Andersson, O.; Tai, F.-I.; Ederth, T.; Liedberg, B. Lateral Control of Protein Adsorption on Charged Polymer Gradients. Langmuir 2009, 25, 3755-3762.

(29) Ekblad, T.; Faxälv, L.; Andersson, O.; Wallmark, N.; Larsson, A.; Lindahl, T. L.; Liedberg, B. Patterned Hydrogels for Controlled Platelet Adhesion from Whole Blood and Plasma. Adv. Funct. Mater. 2010, 20, 2396-2403.

(30) Tai, F.-I.; Sterner, O.; Andersson, O.; Ekblad, T.; Ederth, T. pH-control of the protein resistance of thin hydrogel gradient films. Soft Matter 2014, 10, 5955-5964.

(31) Bozukova, D.; Pagnoulle, C.; De Pauw-Gillet, M.-C.; Ruth, N.; Jerome, R.; Jerome, C. Imparting Antifouling Properties of Poly(2-hydroxyethyl methacrylate) Hydrogels by 
Grafting Poly(oligoethylene glycol methyl ether acrylate). Langmuir 2008, 24, 66496658.

(32) Ekblad, T. et al. Poly(ethylene glycol)-Containing Hydrogel Surfaces for Antifouling Applications in Marine and Freshwater Environments. Biomacromolecules 2008, 9, $2775-2783$.

(33) Acikgoz, S.; Yungevis, H.; Sanyal, A.; Naci Inci, M. Humidity sensing mechanism based on the distance dependent interactions between BODIPY dye molecules and gold thin films. Sens. Actuators, A 2015, 227, 21-30.

(34) Harun, N. I.; Ali, R. M.; Ali, A. M. M.; Yahy, M. Z. A. Resistive-type Humidity Sensor Based on CA-NH $4 \mathrm{BF}_{4}-\mathrm{PEG}_{600}$ Thin Films. Phys. Procedia 2012, 25, 221-226.

(35) Ranucci, E.; Opelli, P.; Ferruti, P.; Ferrari, V.; Taroni, A.; Marioli, D. Poly(ethyleneglycol)s-based hydrogels as coatings for relative humidity sensors. Polym. Gels Networks 1994, 2, 119-133.

(36) Acikgoz, S.; Bilen, B.; Demir, M. M.; Menceloglu, Y. Z.; Skarlatos, Y.; Aktas, G.; Inci, M. N. Use of polyethylene glycol coatings for optical fibre humidity sensing. Opt. Rev. 2008, 15, 84-90.

(37) Lequeux, F.; Talini, L.; Verneuil, E.; Delannoy, G.; Valois, P. Wetting of polymers by their solvents. Eur. Phys. J. E: Soft Matter Biol. Phys. 2016, 39, 1-9.

(38) Mujawar, L. H.; Kuerten, J. G. M.; Siregar, D. P.; van Amerongen, A.; Norde, W. Influence of the relative humidity on the morphology of inkjet printed spots of $\operatorname{IgG}$ on a non-porous substrate. $R S C A d v$. 2014, 4, 19380-19388.

(39) Grafting a hydrogel from a thin layer of spun-coated polystyrene, results in similar hydrogel thicknesses, whether the underlying substrate is silica or gold. Lower polymer 
thicknesses obtained on silane-coated silica could be a result of the silane layer providing fewer attachment sites for polymerization.

(40) Penfold, J. Neutron reflectivity and soft condensed matter. Curr. Opin. Colloid Interface Sci. 2002, 7, 139-147.

(41) Feng, W.; Nieh, M.-P.; Zhu, S.; Harroun, T. A.; Katsaras, J.; Brash, J. L. Characterization of protein resistant, grafted methacrylate polymer layers bearing oligo(ethylene glycol) and phosphorylcholine side chains by neutron reflectometry. Biointerphases 2007, 2, 34-43.

(42) Unsworth, L. D.; Tun, Z.; Sheardown, H.; Brash, J. L. In situ neutron reflectometry investigation of gold-chemisorbed PEO layers of varying chain density: Relationship of layer structure to protein resistance. J. Colloid Interface Sci. 2006, 296, 520-526.

(43) Schneck, E.; Schollier, A.; Halperin, A.; Moulin, M.; Haertlein, M.; Sferrazza, M.; Fragneto, G. Neutron Reflectometry Elucidates Density Profiles of Deuterated Proteins Adsorbed onto Surfaces Displaying Poly(ethylene glycol) Brushes: Evidence for Primary Adsorption. Langmuir 2013, 29, 14178-14187.

(44) Wang, W.; Troll, K.; Kaune, G.; Metwalli, E.; Ruderer, M.; Skrabania, K.; Laschewsky, A.; Roth, S. V.; Papadakis, C. M.; MÃijller-Buschbaum, P. Thin Films of Poly(N-isopropylacrylamide) End-Capped with n-Butyltrithiocarbonate. Macromolecules 2008, 41, 3209-3218.

(45) Wang, W.; Metwalli, E.; Perlich, J.; Papadakis, C. M.; Cubitt, R.; MÃijllerBuschbaum, P. Cyclic Switching of Water Storage in Thin Block Copolymer Films Containing Poly(N-isopropylacrylamide). Macromolecules 2009, 42, 9041-9051.

(46) Wang, W.; Kaune, G.; Perlich, J.; Papadakis, C. M.; Bivigou Koumba, A. M.; Laschewsky, A.; Schlage, K.; RÃúhlsberger, R.; Roth, S. V.; Cubitt, R.; MÃijller- 
Buschbaum, P. Swelling and switching kinetics of gold coated end-capped poly(Nisopropylacrylamide) thin films. Macromolecules 2010, 43, 2444-2452.

(47) Zhong, Q.; Metwalli, E.; Kaune, G.; Rawolle, M.; Bivigou-Koumba, A. M.; Laschewsky, A.; Papadakis, C. M.; Cubitt, R.; Muller-Buschbaum, P. Switching kinetics of thin thermo-responsive hydrogel films of poly(monomethoxy-diethyleneglycolacrylate) probed with in situ neutron reflectivity. Soft Matter 2012, 8, 5241-5249.

(48) Zhong, Q.; Metwalli, E.; Rawolle, M.; Kaune, G.; Bivigou-Koumba, A. M.; Laschewsky, A.; Papadakis, C. M.; Cubitt, R.; Wang, J.; MÃijller-Buschbaum, P. Influence of Hydrophobic Polystyrene Blocks on the Rehydration of Polystyrene-blockpoly(methoxy diethylene glycol acrylate)-block-polystyrene Films Investigated by in Situ Neutron Reflectivity. Macromolecules 2016, 49, 317-326.

(49) Galvin, C. J.; Dimitriou, M. D.; Satija, S. K.; Genzer, J. Swelling of Polyelectrolyte and Polyzwitterion Brushes by Humid Vapors. J. Am. Chem. Soc. 2014, 136, 12737-12745.

(50) Galvin, C. J.; Genzer, J. Swelling of Hydrophilic Polymer Brushes by Water and Alcohol Vapors. Macromolecules 2016, 49, 4316-4329.

(51) Sun, L.; Akgun, B.; Hu, R.; Browning, J. F.; Wu, D. T.; Foster, M. D. Scaling Behavior and Segment Concentration Profile of Densely Grafted Polymer Brushes Swollen in Vapor. Langmuir 2016, 32, 5623-5628.

(52) Köhler, R.; DÃünch, I.; Ott, P.; Laschewsky, A.; Fery, A.; Krastev, R. Neutron Reflectometry Study of Swelling of Polyelectrolyte Multilayers in Water Vapors: Influence of Charge Density of the Polycation. Langmuir 2009, 25, 11576-11585.

(53) Wolff, M.; Zhernenkov, K.; Zabel, H. Neutron reflectometery with ADAM at the ILL: Present status and future perspectives. Thin Solid Films 2007, 515, 5712-5715. 
(54) Deodhar, C.; Soto-Cantu, E.; Uhrig, D.; Bonnesen, P.; Lokitz, B. S.; Ankner, J. F.; Kilbey, S. M. Hydration in Weak Polyelectrolyte Brushes. ACS Macro Lett. 2013, 2, 398-402.

(55) Sanjuan, S.; Perrin, P.; Pantoustier, N.; Tran, Y. Synthesis and Swelling Behavior of pH-Responsive Polybase Brushes. Langmuir 2007, 23, 5769-5778.

(56) Larsson, A.; Liedberg, B. Poly(ethylene glycol) Gradient for Biochip Development. Langmuir 2007, 23, 11319-11325.

(57) Thijs, H. M. L.; Becer, C. R.; Guerrero-Sanchez, C.; Fournier, D.; Hoogenboom, R.; Schubert, U. S. Water uptake of hydrophilic polymers determined by a thermal gravimetric analyzer with a controlled humidity chamber. J. Mater. Chem. 2007, 17, 48644871.

(58) Schuld, N.; Wolf, B. A. In Polymer handbook; Brandrup, J., Immergut, E. H., Grulke, E. A., Eds.; John Wiley: New Jersey, 1999; Vol. 2; pp 247-264.

(59) Merrill, E. W.; Dennison, K. A.; Sung, C. Partitioning and diffusion of solutes in hydrogels of poly(ethylene oxide). Biomaterials 1993, 14, 1117-1126.

(60) Akalp, U.; Chu, S.; Skaalure, S. C.; Bryant, S. J.; Doostan, A.; Vernerey, F. J. Determination of the polymer-solvent interaction parameter for PEG hydrogels in water: Application of a self learning algorithm. Polymer 2015, 66, 135-147.

(61) G. Mikos, A.; A. Peppas, N. Flory interaction parameter ÏĞ for hydrophilic copolymers with water. Biomaterials 1988, 9, 419-423.

(62) Petri, H.; Wolf, B. A. Composition-dependent Flory-Huggins parameters: molecular weight influences at high concentrations. Macromol. Chem. Phys. 1995, 196, 23212333. 
(63) Bekiranov, S.; Bruinsma, R.; Pincus, P. Solution behavior of polyethylene oxide in water as a function of temperature and pressure. Physical Review E 1997, 55, 577-585.

(64) Graham, N. B.; Zulfiqar, M.; Nwachuku, N. E.; Rashid, A. Interaction of poly(ethylene oxide) with solvents: 4 . Interaction of water with poly(ethylene oxide) crosslinked hydrogels. Polymer 1990, 31, 909-916.

(65) Biesalski, M.; RÃijhe, J. Swelling of a Polyelectrolyte Brush in Humid Air. Langmuir 2000, 16, 1943-1950.

(66) Pedersen, J. S.; Sommer, C. In Scattering Methods and the Properties of Polymer Materials; Stribeck, N., Smarsly, B., Eds.; Springer Berlin Heidelberg: Berlin, Heidelberg, 2005; pp 70-78.

(67) Matyjaszewski, K.; Xia, J. Atom Transfer Radical Polymerization. Chem. Rev. 2001, 101, 2921-2990.

(68) Chiefari, J.; Chong, Y. K.; Ercole, F.; Krstina, J.; Jeffery, J.; Le, T. P. T.; Mayadunne, R. T. A.; Meijs, G. F.; Moad, C. L.; Moad, G.; Rizzardo, E.; Thang, S. H. Living Free-Radical Polymerization by Reversible Addition-Fragmentation Chain Transfer:The RAFT Process. Macromolecules 1998, 31, 5559-5562.

(69) Wibisono, Y.; Yandi, W.; Golabi, M.; Nugraha, R.; Cornelissen, E.; Kemperman, A. J. B.; Ederth, T.; Nijmeijer, K. Hydrogel-coated feed spacers in two-phase flow cleaning in spiral wound membrane elements: A novel platform for eco-friendly biofouling mitigation. Water Res. 2015, 71, 171-186. 
Graphical TOC Entry

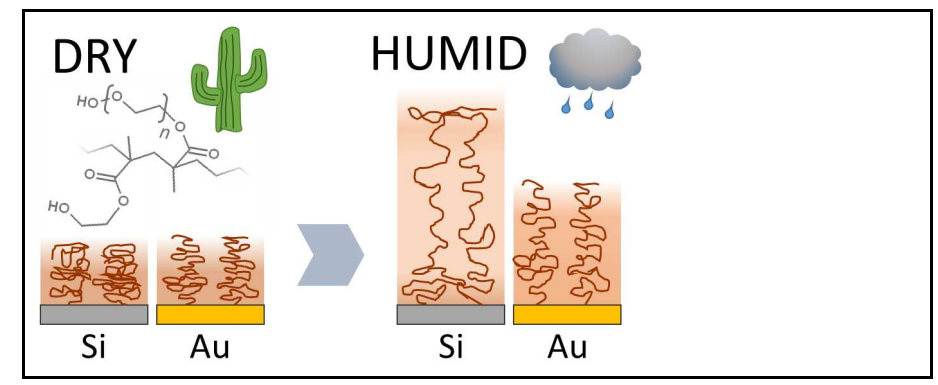




\title{
Supporting Information
}

\section{for}

\section{Swelling of thin poly(ethylene}

\section{glycol)-containing hydrogel films in water}

\section{vapour - A neutron reflectivity study}

\author{
Thomas Ederth ${ }^{*, \dagger}$ and Tobias Ekblad ${ }^{\dagger, \ddagger}$ \\ $\dagger$ Division of Molecular Physics, Department of Physics, Chemistry and Biology, Linköping \\ University, SE-581 83 Linköping, Sweden \\ $\ddagger$ Present address: MariboHilleshög Research AB, Landskrona, Sweden \\ E-mail: ted@ifm.liu.se
}

DOI: 10.1021/acs.langmuir.8b00177 


\section{Fitting of the neutron reflectivity data}

\section{Notes on the fits in Figure $4 \mathrm{a}$}

The general approach adopted for the fitting was to determine the properties of the substrates before coating with the polymer, to obtain the substrate parameters (thicknesses, SLDs and roughnesses) and then to keep these fixed in the subsequent fitting of the polymer layers.

For the polymer layers, we have tried to keep parameters consistent between the different humidities (and also the different water contrasts, for the data in water). We aimed at minimizing the number of fit parameters, and at using the simplest model that still produces acceptable fits to the data. In practice, this often results in occasional calculated profiles that do not fit the data perfectly. However, the best global fit result is preferred over the introduction of 'ad hoc' layers or parameters to improve the fits for individual curves. Additional layers were necessary to explain the distribution of polymer at higher humidities, but adding or changing fit parameters that do not relate to the polymer layer have been avoided. There are some deviations between the fits and the data, which are relatively small considering the overall agreement, but which deserve a comment.

The deviation at low $q$ for the lowest humidities, i.e. at the drop near the critical edge, cannot be resolved by physically meaningful additional layers, or a more elaborate model of the polymer. The difference between the measured and fitted critical edge suggest that the actual SLD difference between the substrate and the bulk is greater than what the model accounts for. Indeed, the deviation can be resolved by assigning a bulk (air) SLD of $-0.4 \times 10^{-6} \AA^{-2}$, (thus increasing the difference from the silicon SLD) with very small adjustments of other parameters. This is not a physically meaningful change, but it is by far the smallest change in the overall set of parameters that resolves the deviation. We believe that the discrepancy between the fit and the data here is the result of an error emanating from the over-illumination of the sample at the lowest $q$, i.e. the lowest angles, where the beam illuminates an area larger than the sample, and the subsequent correction 
of the reflectivity in the normalization procedure to take into account how large portion of the beam actually hits the sample. The deviation could also be addressed by using the angle of incidence on the sample as a fit parameter (tested with GenX), and small adjustments to this will also account for the difference between data and model. However, we believe that it is more honest, and also more meaningful for the interpretation not to do this, but rather to minimize the number of fit parameters.

The deviation for the $98 \% \mathrm{RH}$ i the $q$ range $0.02-0.03$ is most likely a result of a slight change in humidity due to drift in the humidity control. At the highest humidities, the humidity control was slower in responding to, and in correcting, deviations. This appears to have resulted in a slight variation in thickness over the course of the experiment. Since data is collected from low to high $q$, stabilization of the humidity level after some time results in fringes at high $q$ being well resolved, but not those at low $q$. However, that the oscillations in the data are not as well resolved as in the fit, could also be caused by roughness, or other inhomogeneities developing upon swelling.

The above issues can be 'resolved' by introducing additional fit parameters, but we have been unable to improve the fits using physically meaningful changes in the model for the polymer layer, and hence prefer to present the data as they are. Taken as a whole, we find the data to be consistent with the used models.

\section{The choice of polymer model}

The additional layers introduced for the highest humidities for the polymer on the silicon substrate result in significant improvements of the fits. A comparison of one-, two- and three-layer models is shown in Figure S1, with corresponding parameters included in the table below. The fitting procedure takes the data errors into account, and thus deviations at high reflectivity (low $q$ ) are given greater weight. For each value of the RH, models with additional layers were tested, in addition to those presented in the main text, but where the this did not result in improved fits, they were discarded. 
Table S1: Parameters for the optimized models with different numbers of layers for the sample on silicon at $98 \%$ RH. Layers are numbered from the silicon substrate and outwards.

\begin{tabular}{lccccc}
\hline Model & Layer & $\begin{array}{c}d \\
(\AA)\end{array}$ & $\begin{array}{c}\text { SLD } \\
\left(\times 10^{-6} \AA^{-2}\right)\end{array}$ & $\begin{array}{c}\sigma \\
(\AA)\end{array}$ & $\chi^{2}$ \\
\hline 1-layer & 1 & 435 & 2.71 & 7 & 0.0670 \\
2-layer & 1 & 256 & 2.45 & 42 & 0.0533 \\
& 2 & 163 & 2.80 & 6 & \\
3-layer & 1 & 57 & 2.41 & 28 & 0.0276 \\
& 2 & 306 & 3.04 & 11 & \\
& 3 & 58 & 2.89 & 8 & \\
4-layer & All tested 4-layer models give & $\geq 0.0273$ \\
\hline
\end{tabular}

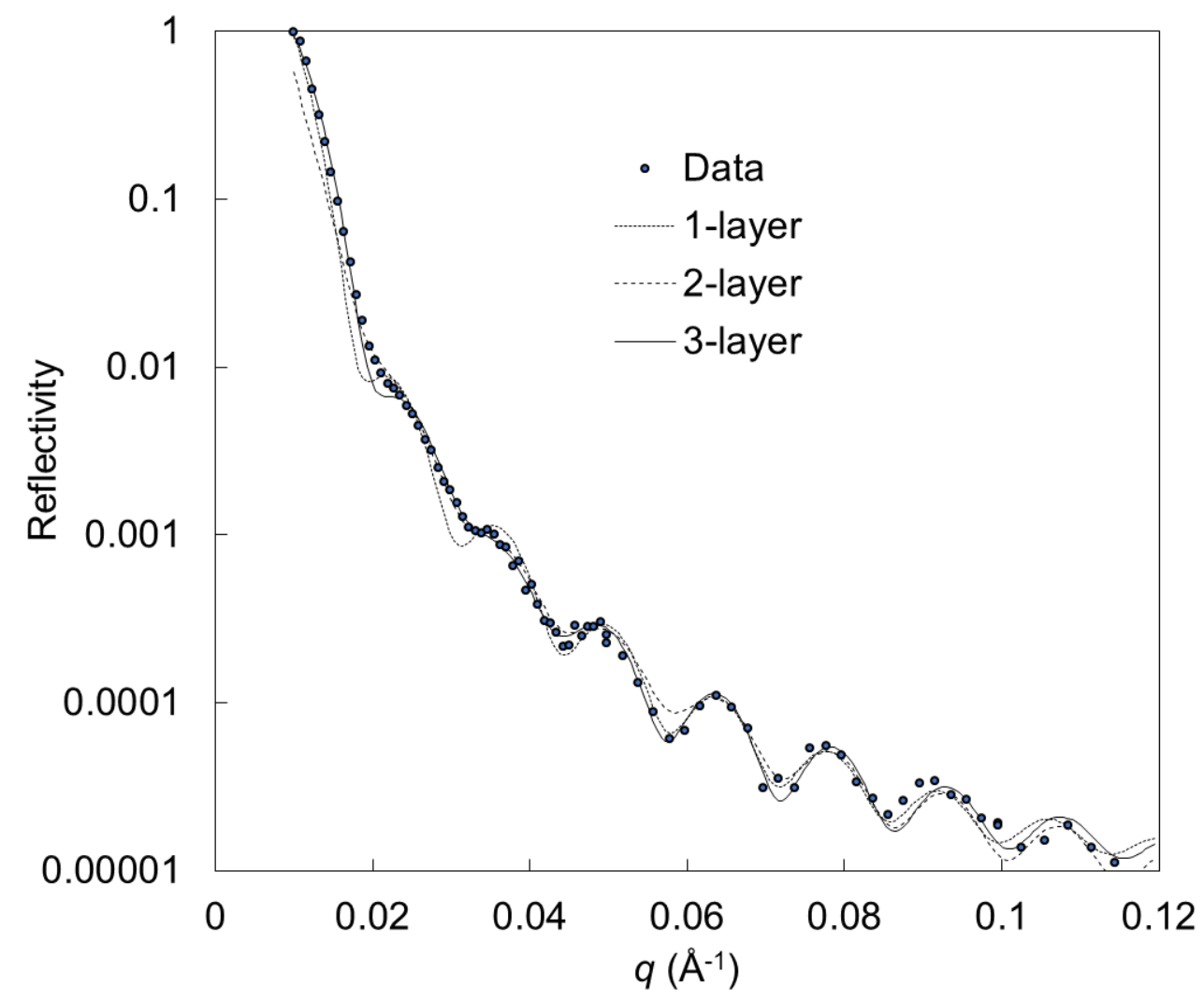

Figure S1: The data for the polymer on silicon at $98 \% \mathrm{RH}$, shown with calculated reflectivities for the optimized models using one, two and three layers, respectively. 


\section{Water volume fraction profiles}

In addition to the polymer volume profiles displayed in Figures $5 \mathrm{~b}$ and $6 \mathrm{~b}$ in the main text, the corresponding water volume profiles are included here, for clairity. These are obtained via $\phi_{w}=1-\phi_{p}$, where $\phi_{w}$ and $\phi_{p}$ are the volume fractions for water and polymer, respectively.
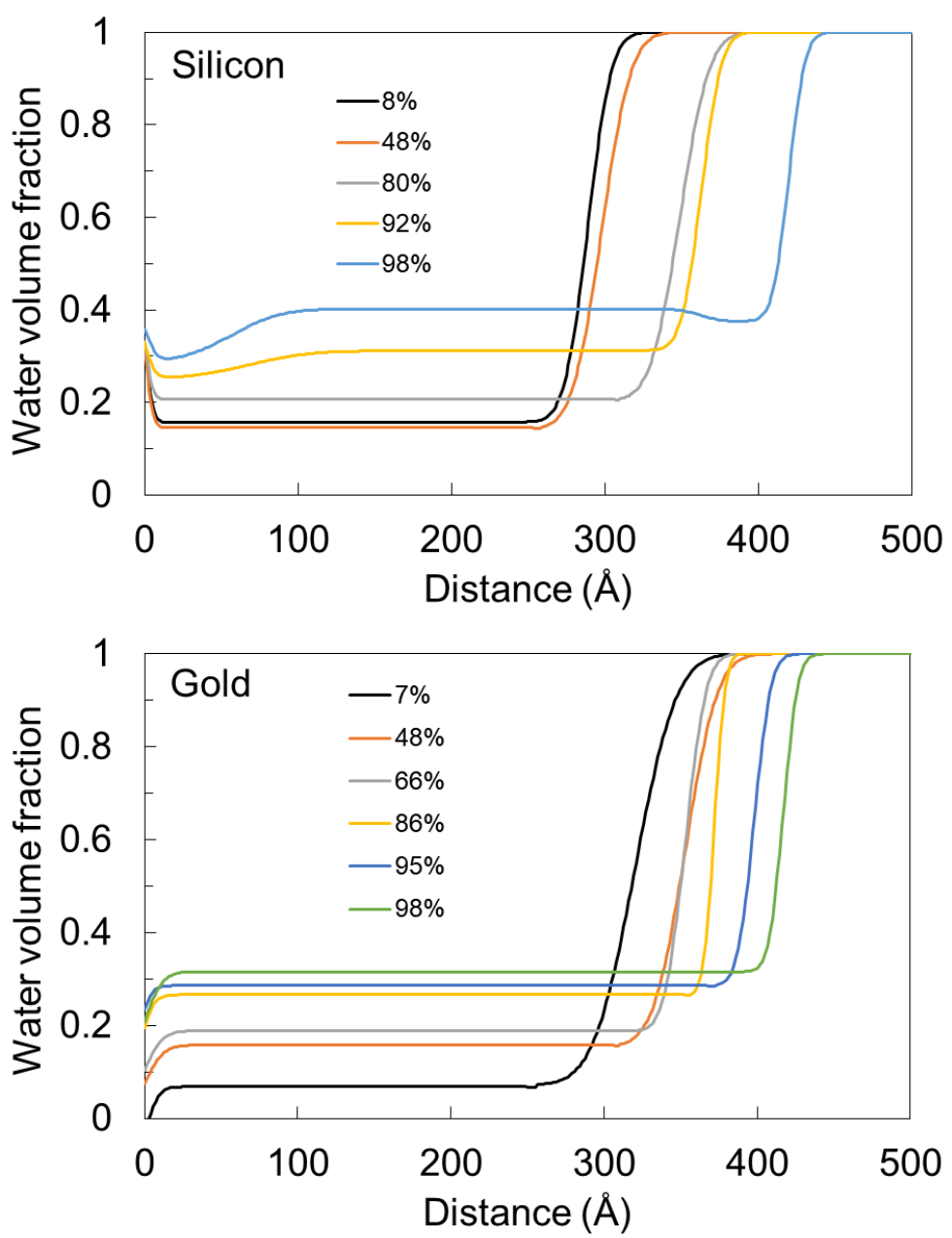

Figure S 2: Water volume fraction profiles for the two substrates. Note that the changes in the profile over the first $20 \mathrm{~nm}$ are artefacts caused by the assigned interfacial roughness of the solid-liquid interfaces. 


\section{Reflectance of silicon and gold substrates}

We investigated whether the faster polymerization rate on gold could be explained by reflection of the incident UV light from the gold layer, and in this way increasing the effective photon density in the film. For this, the reflectance of both Si wafers (with a native oxide layer) and gold-coated samples was measured in a Shimadzu UV-2450 UV-Vis Spectrophotometer, with a custom-built specular reflectance attachment, measuring the reflectance at approximately $5^{\circ}$ from the surface normal. Baseline correction of the spectrometer was made using an aluminium mirror as the sample, and the relative reflectance of the Si and Au substrates were recorded, see Figure S3. The ratio of the reflectance from the silicon surface $\left(R_{\mathrm{Si}}\right)$ to that from the gold surface $\left(\mathrm{R}_{\mathrm{Au}}\right)$ was calculated and is shown in the lower part of Figure S3. The ratio shows that the reflectance from the Si surface is greater than the reflectance from the gold surface over the whole measured UV range, i.e. from $200 \mathrm{~nm}$ and upward. The used UV lamp (Philips TUV PL-L, 18W) has its main emission peak at 254 $\mathrm{nm}$, and smaller emission peaks at higher wavelengths. Thus, the faster polymerization on gold cannot be explained by a higher light flux due to reflection from the substrate.

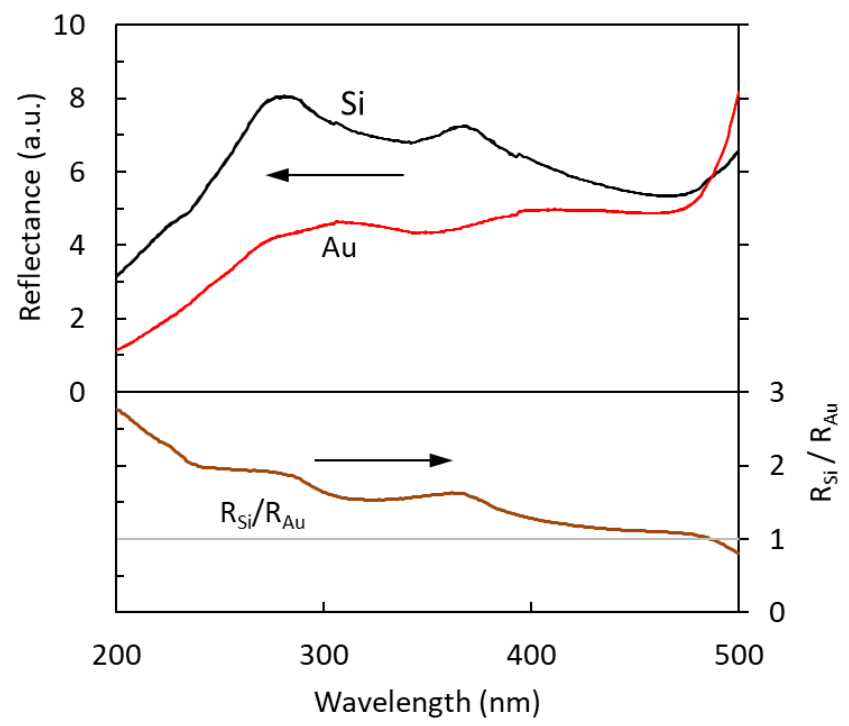

Figure S 3: Top: relative reflectivities of silicon and gold substrates. Bottom: The ratio of the two reflectivites shows that the reflectivity is higher for UV light from the silicon substrate, over the whole investigated UV range. 